\title{
Jenseits von Pathos und Verwertungspragmatismus
}

Bericht vom 34. Kongress der Deutschen Gesellschaft für Soziologie

6.-10. Oktober 2008 in Jena

\section{Von Paula-Irene Villa}

Auch in Jena wurde - nicht nur, aber vor allem in den wahrscheinlich wichtigsten Veranstaltungen aller Soziologiekongresse, nämlich bei informellen Gesprächen auf den Fluren, beim Kaffee, in den Kneipen und an den Bücher- und Projektständen - viel über die globale Finanzkrise gesprochen. Während die deutsche wie die weltweite Politik Anfang Oktober 2008 angesichts der Dramatik von Bankenpleiten, implodierenden Immobilienmärkten und einer drohenden Rezession zwischen Schock und Aktivismus schwankt, wendet sich die Soziologie der behutsamen Reflexion, gar der Selbstreflexion zu. Das allein ist nicht nur im Lichte politischer wie medialer Schnellschüsse immer gut, sondern auch vor dem Hintergrund des Faches so wichtig wie wohltuend: Chronisch hat sich die Soziologie in den vergangenen Jahren (Jahrzehnten?) selbst obsolet gewähnt, weil sie keine Rezepte für gesellschaftliche Probleme anbietet, weil sie einige gravierende soziale Transformationen nicht vorausgesehen hat und / oder weil sie sich schon lange nicht mehr auf einen Nenner und damit nicht auf den einen öffentlichkeitswirksamen Begriff bringen lässt. Und oft ist die Soziologie in der Öffentlichkeit für ihre Zurückhaltung - die sicher auch etwas mit bisweilen sterilen Theorie- und Methodendiskussionen oder mit manchmal obskur scheinenden Gegenständen zu tun hat - für irrelevant erklärt worden. Doch die Soziologie, so schien es mir als natürlich partielle Beobachterin des diesjährigen Kongresses, ficht das nicht (mehr) sonderlich an. Sie hat sich, wohl wissend um ihre Stärken, ihre Grenzen sowie um ihre Aufgaben der behutsamen, differenzierten und multiparadigmatischen Reflexion zugewandt. Die Soziologie weiß, dass diese Reflexion ansetzen muss an den auch tagespolitisch drängenden sozialen Fragen. So waren in Jena beispielsweise „Prekarisierungsprozesse' ein intensiv diskutiertes Problem. Zugleich weiß die Soziologie, dass diese Reflexion nur in analytischer Distanz zur Logik des Tagesgeschäfts in Politik und Medien funktioniert, zumindest wenn sie mehr bieten soll als wohlfeile, bloß überlegt klingende Rhetorik. Soziologische Reflexion impliziert nicht nur einen nüchternen, zugleich neugierigen und Anteil nehmenden Blick auf die (unsichere) soziale Welt - soziologische Reflexion auf die Gegenwart impliziert zudem den kritischen, fragenden Blick auf die eigenen Kategorien, mit denen wir uns einen Reim auf die „unsicheren Zeiten“ machen.

Diese positive Einschätzung ist ganz sicher eine partielle: zum einen, weil es (trivial, aber entscheidend) unmöglich ist, sich einen Gesamteindruck des Kongresses im engeren Sinne zu verschaffen: Bei neun Plenar- und 35 Sektionsveranstaltungen, knapp 50 ad hoc-Gruppen, allerlei Verlagsempfängen und sonstigen Angeboten - von Konzerten über Poster Sessions bis Stadtführungen - und knapp 2000 Teilnehmenden ist die bloße physische Anwesenheit eine ebenso voraussetzungs- wie folgenreiche inhaltliche Entscheidung. Zum anderen rührt meine Einschätzung aus der eigenen Verortung im Fach: Die Beschäftigung mit der geschlechtlichen Dimension des Sozialen nötigt seit jeher zu einer besonderen Reflexivität, denn sie sucht unter anderem nach der Wirksamkeit auch unausgewiesener Normen und Strukturen, sie bewegt sich im Spannungsfeld zwischen politischen Herausforderungen und theoretisch wie methodisch tückischer ,Mühsal der Ebenen', sie bürstet das angeblich allgemeine Fachwissen - ,die Arbeit', ,die Moderne', ,die Prekarisierung', ,die Unsicherheit' usw. - systematisch gegen den androzentrischen Strich. Das muss nicht bedeuten, immer und überall nach dem Geschlecht zu suchen (obwohl auch dies sinnvoll ist). Doch bedeutet es immer zu fragen, ob wirklich und für wen in welcher Weise das drin ist, was auf soziologischen Diagnosen, Analysen und Theoretisierungen drauf steht. Kurzum: Gender Studies sind gerade in ihrer soziologischen Version 
„paradigmatische Verunsicherungswissenschaften“ (Degele 2003). Durch diese zwei Justierungen meiner Brille stellte sich Jena, durchaus im Unterschied zu anderen DGS-Kongressen, als guter Kongress dar.

Allerdings gab es auch unfassbar schlechte Veranstaltungen, die der reflexiven und damit produktiven Verunsicherung nicht nur nicht gerecht wurden, sondern genau das Gegenteil präsentierten. Zu dieser gehörte - leider! - die Abschlussveranstaltung zum an sich interessant gewählten Thema „Neue Unsicherheiten - Männer auf verlorenem Posten“. Was sich die Organisatorin, eine in der Geschlechterforschung bestens ausgewiesene Soziologin, bei der $\mathrm{Zu}-$ sammenstellung des Podiums gedacht haben mag, ist zwar leicht erkennbar (Kontroverse), aber gründlich missraten: Auf dem Podium schwadronierte die Psychiaterin und klinische Psychoanalytikerin Hanna Ziegert in brachialstem Krisen-Duktus über die ,Eigentlichkeit' des Geschlechts, das irgendwo zwischen Trieben, Genen und frühkindlichen Erfahrungen liegen sollte, denunzierte sozialwissenschaftliche Perspektiven und kanzelte alle Hinweise auf entsprechende Differenzierungen mit dem Hinweis auf die eigene Erfahrung in der forensischen Praxis ab. Dabei verstieg sie sich zu mehr als gewagten Thesen bezüglich sexualisierter Gewalt - die Mütter der Männer sind schuld! - und ließ keinerlei Dialogfähigkeit oder auch nur Reflexionsvermögen in ihrer anekdotischen Katastrophenrhetorik erkennen. Auch dann nicht, als der Historiker Jürgen Martschukat sie als Repräsentantin eines Krisendiskurses vorführte, den er in seinen historischen Forschungen zu Männlichkeiten kritisch analysiert (vgl. Martschukat / Stieglitz 2007). Christel Eckart, irritierenderweise die einzige Soziologin auf dem Podium, betonte die gesellschaftliche Notwendigkeit einer Aufwertung von ,care'-Tätigkeiten und behauptete, die derzeitige Forcierung von Fürsorgearbeit (etwa die Zumutungen , aktiver Vaterschaft') verunsichere die Männer. Darüber ließe sich streiten, dazu gibt es nunmehr reichlich soziologische Reflexion und Forschung (vgl. Villa 2006). Doch das war alles bei dieser Diskussion nicht präsent. Vielmehr war das Niveau der Debatte so tief, dass es schlicht unfassbar war. Kontroversen zu Männlichkeit im Lichte der Verunsicherung? Gern. Aber auf einem Soziologiekongress doch bitte auch bei diesem Thema angemessen differenziert und auf dem Stand der soziologischen Reflexion bzw. Forschung.

Von solchen Ausrutschern aber abgesehen, führten tatsächlich eine Vielzahl von Beiträgen und Veranstaltungen nicht nur Fragezeichen im Titel, sondern behielten einen unprätentiösen, nachdenklichen Duktus bei.

\section{Trans- oder inter- oder doch national?}

Ulrich Beck hat in seinem Eröffnungsvortrag (erneut) nachdrücklich auf die Notwendigkeit hingewiesen, den bislang nach wie vor nationalen Blick der in Deutschland betriebenen Soziologie durch eine transnationale Brille zu erweitern. Entlang dreier, aus seinen bisherigen Arbeiten wohl bekannten Entgrenzungs-Thesen (1) Entgrenzung sozialer Ungleichheit, (2) Entgrenzung sozialer Gleichheit und (3) Entgrenzung von Natur und Gesellschaft) plädierte er - wiederholt - für eine „Neujustierung“ der Soziologie, etwa im Sinne einer „Neuvermessung sozialer Ungleichheit“ und der soziologischen Reflexion auf die Historizität von Ungleichheitskategorien. Das ist alles nicht neu, doch angesichts des weiterhin hegemonialen Nationalismus der deutschsprachigen Soziologie sicher notwendig. Denn auch der Jenaer Kongress war weit davon entfernt, diese nationalstaatliche Fixierung zu überwinden. Die Vielzahl an sich interessanter und wichtiger Vorträge in national vergleichender Perspektive (,xy in USA, Deutschland und Schweden', ,yz: Frankreich und Deutschland im Vergleich' usf.) ist hierfür ebenso ein Beispiel wie die Ablehnung einer ad hoc-Gruppe zur ,postkolonialen 
Soziologie'. ${ }^{1}$ So präsentiert sich das Fach, jedenfalls in weiten Teilen, einer nationalen Perspektive verhaftet, die sich in der Form inter-nationaler Vergleiche nur verdoppelt. Dabei ist allerdings eine ,Vermainstreamung' und damit Normalisierung von Reflexionen auf nichtdeutsche, gar außereuropäischen Regionen zu beobachten, die ganz analog zur Vermainstreamung der Gender-Dimension verläuft: Im Plenum zur „Gemeinschaftsbildung als Modus der Unsicherheitsabsorption“ z.B. wurden, insgesamt recht bunt und ohne erkennbaren inneren Zusammenhang, „Netzwerk-Gesellschaften“ (Boris Holzer) am Beispiel Chinas und Russlands analysiert oder am Beispiel ,,indigenes Mexico“ (Wolfgang Gabbert) der Zusammenhang zwischen „lokalen und imaginierten Gemeinschaften“ diskutiert. Solche Vortrags-Zusammenstellungen, die auch die Frage nach ,posttraditionaler Vergemeinschaftung“ (Hitzler / Pfadenhauer) im Modus des Konsums (in Deutschland), die Verknüpfung von ,,persönlicher Unsicherheit mit der Einbettung in gemeinschaftliche Strukturen“(Bühler) sowie den „Security-Insecurity-Ansatz“ am Beispiel des „Darmstädter Community Survey“ der Nachkriegsjahre (Gerhard / Arnold) zusammenschnürten, zeigen, dass eben unter einem vagen thematischen Dach nebeneinander über Darmstadt, Mexico, China und Russland gesprochen werden kann. Zu begrüßen ist das im Prinzip; das Nicht-Deutsche wird nicht mehr in die Parallelveranstaltungen abgedrängt, nicht mehr eingehegt in die Migrations- bzw. so genannte Entwicklungssoziologie oder in die ausgewiesenen, Gastland'-Veranstaltungen. ${ }^{2}$ Aber zueinander finden diese regional weit voneinander entfernten Beiträge deshalb nicht, weil sie eben national gerahmt sind. Inter- statt transnational. In solchen thematisch ,internationalen' Veranstaltungen, und davon gab es einige, wird deutlich: Deutschland, allenfalls noch die Schweiz und Österreich werden differenziert diskutiert. Und sie werden eben nicht als nationalstaatlich verfasste Gesellschaft unter anderen ausgewiesen - man kann sich nach wie vor sicher sein, dass wenn keine nationale Markierung drauf steht, Deutschland drin ist. Wenn es um , andere Länder’ oder Regionen geht, wird es hingegen schnell pauschal: „Chinas Jugend vor den Herausforderungen ihres Landes“ (Jonda) beispielsweise. Mal abgesehen davon, dass ,ihr Land“ doch ziemlich groß, vielfältig und in sich widersprüchlich ist und es ,die Jugend“ auch in China schon lange nicht gibt, mache man das Gedankenspiel: „Deutschlands Jugend vor den Herausforderungen ihres Landes“. Klingt das auf der Höhe der Soziologie? Eben.

Aber es ging auch anders: Dallinger / Eichler diskutierten ein sozialpolitisches Thema „der graue Markt der Altenpflege“ - in einer neuartigen Perspektive, eben der ,transnationalen“, und verwiesen dabei auf die systematische Relevanz von transnationalen (Pendel-)Migrationen nicht nur für sozialpolitische Systeme und für spezifische Dienstleistungsmärkte, sondern auch für das konkrete Zusammenleben in Familien. Leider wurde hier kaum Bezug genommen auf einschlägige Studien und Einsichten aus der intersektionalen Geschlechterforschung zum „Weltmarkt Privathaushalt“ (vgl. etwa Gather / Geissler / Rerrich 2007). Denn gerade der transnationalisierte Pflegebereich lässt sich ohne eine systematische Bezugnahme auf vergeschlechtlichte Arbeitsteilungen im globalen Maßstab wohl kaum verstehen. Transnational argumentierte (wie zu erwarten) auch Beck-Gernsheim in ihrem Beitrag zu ebensolchen „Heiratsmärkten“ im Kontext zunehmend restriktiver Migrationsregime. Auch die erste Sektionsveranstaltung der Sektion Frauen- und Geschlechterforschung thematisierte, in gewohnt reflexiver Manier, die „ÜberKreuzungen“ (Klinger / Knapp 2008) verschiedener „Achsen der Ungleichheit“ (Klinger / Knapp / Sauer 2007) anhand des Oberthemas „Unsicherheiten

1 Überhaupt wurde auch beim diesjährigen Kongress wieder auf den Fluren der Unmut über die mangelnde Transparenz bei der Auswahl von ad hoc-Gruppen wenn nicht laut, so doch hörbar formuliert. Allen ist klar, dass eine Auswahl nötig ist - der DGS-Kongress gleicht sowieso schon mehr einer Messe als einer Fachveranstaltung. Doch transparente Kriterien bei der Auswahl und eine klarere Strukturierung der ad hoc-Gruppen, auch in der Vorbereitungsphase, sind gerade angesichts der Menge notwendig.

2 Die diesjährigen Gastländer waren Polen, Tschechien und Ungarn. 
und Geschlechterordnungen in transnationalen Räumen“. In den Beiträgen wurde deutlich, wie wenig auch empirisch plausibel die Analyse nur einer Dimension - Geschlecht oder Transnationalität - ist: „Mehrebenenanalytische Betrachtungen“ (Heidemarie Winkel) sind nötig, die „Verhältnisse zwischen Wohlfahrts-, Migrations- und Geschlechterregimes“ sind selbst „,prekär" (Anja Weckwert) und lokale Governanceprozesse der Ressourcenpolitik verschränken sich mit globalen (Christine Bauhardt).

\section{Unsicherheit: Prekarisierung de Luxe?}

Das zentrale Stichwort des Jenaer Kongresses war Unsicherheit. Dieses ebenso vage wie ins Schwarze der Gegenwart treffende Motto wurde lebendig in den vielen Beiträgen und Diskussionen, die sich explizit der Unsicherheit annahmen - die semantische Nötigung der DGS forderte alle heraus, sich nicht nur, wie eingangs angedeutet, mit sich und den eigenen Begrifflichkeiten auseinanderzusetzen, sondern vor allem nach Unsicherheiten in der sozialen Wirklichkeit Ausschau zu halten. Und man stellt fest: In dieser stellt sich Unsicherheit oft drastischer dar, nämlich als Prekarisierung. Der Sozialstaat ist national wie international massiv im Wandel (vgl. Lessenich 2008: 9-20), mit gravierenden sozialen Folgen für die Menschen. Dass entsprechende sozialpolitische Fragen wie die nach Verteilungs(un)gerechtigkeiten - die neue „soziale Frage am Beginn des 21. Jahrhunderts“ (Dörre 2008) - und damit verbundenen gesellschaftstheoretischen wie -analytischen Dimensionen wieder in den Vordergrund der soziologischen Aufmerksamkeit rücken und auch beim diesjährigen Kongress so präsent waren, ist neben der ökonomischen Krise sicher auch den lokalen Organisatoren und Organisatorinnen geschuldet: Die Jenaer Soziologie profiliert die Analyse der „Neuerfindung des Sozialen“ (Lessenich 2008) aus dem Geist des „flexiblen Kapitalismus“ sowie anhand der Auseinandersetzung mit den (verunsichernden, weil enorm beschleunigten) zeitlichen Logiken der Moderne (vgl. Rosa 2005).

Angesichts der drohenden Rezession und der etablierten Normalität von Hartz IV inklusive der damit verbundenen Neu- und Deregulierungen in sozialpolitischer Hinsicht, nach einer intensiven soziologischen Debatte um Inklusion / Exklusion (vgl. exemplarisch Bude / Willisch 2007) usw. ist sämtliche Romantik perdu: Die Entgrenzung von Leben und Arbeit, das ,home-office', die räumlichen ultramobilen Pendeleien zwischen befristeten Kreativjobs und die ,Arbeit am Selbst' (,fit for success') schillern nicht mehr als Ausdruck avantgardistischer Lebensführung in der post-industriellen Wissensgesellschaft. Vielmehr hat sich die Soziologie - wieder? - den im engeren Sinne Problemen weiter Teile der (deutschen wie europäischen und gar Welt-) Gesellschaft angenommen, die sich, nach wie vor, vor allem aus dem Sein (durchaus à la Marx) ergeben. Das große Interesse an diesen Problemen zeigte etwa der Andrang beim Forum mit Günter Wallraff zum Thema „Unsichere Arbeitswelt“" - den die Autorin aber eben aufgrund der Menschenmassen nicht besuchen konnte. Interessanterweise blieb es in den vielen Beiträgen, die sich mit dem ,Sein' und seinen Verunsicherungen auseinandersetzten, nicht bei einer bloßen Analyse der Wucht der prekarisierenden Verhältnisse. Diese im Blick behaltend, machten sich viele Kollegen und Kolleginnen auf, nach den Akteuren und Akteurinnen zu schauen. Wie gehen also Menschen konkret mit den verunsichernden Zeiten um? Erleben sie sich als Opfer? Zeigen sich neue kreative Eigensinnigkeiten und Handlungsmächtigkeiten?

Irene Dölling wies in ihrer Mittagsvorlesung unter dem Titel „Ent-Sicherung in Zeiten gesellschaftlicher Transformationen“ auf die Notwendigkeit hin, „,das Handeln“ der Menschen in den Blick zu nehmen. Ihr Vortrag war auch deshalb so erhellend, weil er die Ungleichzeitigkeiten der deutschen Wirklichkeit aus der doppelten Perspektive von Ost / West und Männern / Frauen auslotete. Mit einem an Bourdieu orientierten Zugriff machte sie darauf aufmerksam, dass der derzeitige Subjektivierungsdiskurs - wie er etwa in der aktuellen Gouver- 
nementalitätsdebatte, in vielen post-strukturalistischen und diskurstheoretischen Zugängen (vgl. Bröckling 2007) formuliert wird - Gefahr läuft, reale Individuen in ihrer je empirischen Wirklichkeit auszublenden. Der ,praktische Sinn' verschwindet dabei unter der Beschreibung der Verhältnisse (seien diese diskursiv, ökonomisch oder beides zugleich); die „,praxeologische Erkenntnis“ (Bourdieu 1976: 148) bildet sich in der Soziologie dann nicht mehr ab, sondern verkommt zu einer bloßen Inkorporierung sozialer Strukturen. Und das ist weder im Sinne Bourdieus noch der sozialen Wirklichkeit angemessen. Mit solch einer Verkürzung in der Analyse läuft, so Dölling abschließend, die Soziologie Gefahr, doxische Einstellungen zu perpetuieren. Wichtig wäre es demgegenüber, den praktischen Sinn auch empirisch zu rekonstruieren. Keine neue Forderung - aber nach wie vor ein wesentliches Desiderat. Aufgegriffen wurde dies teilweise in einer Sektionsveranstaltung der Sektion Frauen- und Geschlechterforschung unter dem Titel „Wandel der Sozial- und Geschlechterordnung durch Prekarisierung“. Trinkhaus et al. stellten in ihrem Beitrag ein empirisches Projekt vor, das in Brandenburg der prekären Kategorie ,Männlichkeit' nachgegangen ist, ohne direkt nach dieser zu fragen. Das Originelle am präsentierten qualitativen Forschungsdesign war der Einsatz von Collagen als Mittel, mit dem junge Frauen und Männer ihre im wörtlichen Sinne Bilder von Männlichkeit zu Papier brachten. Den jungen Menschen war klar, so ein Ergebnis, dass die Zukunft hochgradig unsicher ist und dass auch ihr Handeln darin keinem klaren Pfad folgen kann. Dies gilt für vieles, auch für ihre geschlechtliche Identität. In den Collagen, so wurde anschaulich, bildeten sich konstitutive strukturelle Rahmungen des Handelns ebenso ab wie eigene Sehnsüchte, Planungen und Handlungsspielräume der Konstruktion. Auf andere Weise griffen Susanne Völker und Sabine Hark in ihrem Beitrag die Stichworte Wandel und Prekarisierung auf, nämlich als Reflexionsherausforderung für das Fach in seiner Begrifflichkeit und Methodik: Sie stellten fest, dass die Gesellschaftsdiagnose von Des-Integration und Entsicherung einerseits in ihrer Problemdiagnose plausibel sei, andererseits aber auf der Ebene der soziologischen Semantik Gefahr laufe, die ,Ausgeschlossenen', ,Exkludierten', ,Unsichtbaren', ,Überflüssigen' in unzulässiger Weise zu verobjektivieren. Das heißt, das ,neue Prekariat' wird - liest man entsprechende Beschreibungen diskursanalytisch näher und auch methodisch fundiert zwischen den Zeilen - reduziert auf eine geradezu , asoziale' Existenz, ein bloß somatisches Sein. Wiederum mit Bourdieu plädierten die Vortragenden dafür, einen praxeologischen Blick auf das ,doing precarity“ zu richten. Die Begründung hierfür ist so einfach wie bestechend: Soziale Praxis folgt ihrer eigenen Logik, nicht derjenigen der soziologischen Begrifflichkeit. Und diese Praxis ist, so die Autorinnen, mehr denn je von Unbestimmtheit konstituiert. Bisherige Geschlechterordnungen - wie das mehr oder minder kohärente Ineinandergreifen von Erwerbsarbeit, Männlichkeit, Öffentlichkeit bzw. Familie, Weiblichkeit, Privatheit - sind keineswegs im Strudel post-fordistischer Transformationsprozesse verschwunden, doch haben sie sich gehörig gewandelt. Vor allem die Transformation von ,Arbeit', dessen Überhöhung einerseits (etwa in den familienpolitischen Instrumenten zur Inklusion von Müttern auf den Erwerbssektor) und gleichzeitiger Bedeutungsverlust bzw. Neukodierung (Stichwort Entgrenzung) verunsichern die Praxis zutiefst. Um die Rekonstruktion dieser Verunsicherung müsste es gehen, so Völker und Hark, nicht um die begriffliche Vereindeutigung, die ja im Effekt Sicherheiten schafft. Anders gesagt: Die Soziologie sollte nicht durch die Formulierung vermeintlicher Sicherheiten - ,DIE Unsicherheit' - an der Zementierung der Verhältnisse mitwirken.

\section{Unsicherheiten allerorten}

Genau dieses Problem wurde in einer interessanten Veranstaltung zum Forschungsrating diskutiert. Nachdem Friedhelm Neidhardt die Ergebnisse der Studie des Wissenschaftsrats (vgl. Wissenschaftsrat 2008) gegen Kritiker/innen verteidigte und dabei insbesondere auf die methodischen Aspekte abhob, formulierte Trutz von Trotha mit dem ihm eigenen Galgenhumor 
und sicherlich stellvertretend für viele einige Kritikpunkte. Das Problem, dass ein Forschungsrating nicht anders kann, als Reflexion und Forschung zu operationalisieren und damit alles andere als rein deskriptiv vorgeht (sondern auch präskriptiv definiert, was gute Forschung ist), bleibt bestehen. Die Inkommensurabilität von Operationalisierung einerseits und intellektueller Reflexivität andererseits, die Unvereinbarkeit von wettbewerbsorientierter Managementlogik und soziologischer Kreativität ist und bleibt eine Spannung, die sich wohl nur im Einzelfall und mit wiederum gesteigerter Reflexivität meistern lässt. Hans-Georg Soeffner, derzeitiger DGS-Vorsitzender, betonte jedenfalls bei dieser Diskussion, dass es zwar eine Illusion sei, als Fachgesellschaft ,das Ganze' zu kontrollieren, dass aber nur ein intensives Engagement der DGS bzw. aller Institute und KollegInnen dafür garantieren könne, dass die Rankingatoren (eigene Formulierung!) wissen (könnten), was sie tun. Dazu gehört auch eine stärkere Beforschung der Effekte solcher Ratings in der Öffentlichkeit. Nicht zuletzt im Vorgriff auf die demnächst bevorstehende zweite Exzellenzrunde ist dies eine wichtige Aufgabe. Die Hochschulen bereiten sich hierauf schon vor und die Sozialwissenschaften sollen, so wird jedenfalls mancherorts gemunkelt, verstärkt dabei sein. In meiner (zum Teil leidvollen) Erfahrung ist nur ein offensiver Umgang mit technokratischen Logiken sinnvoll, d.h. der Versuch einer Quadratur des Kreises - meinetwegen etwa ein MA in ,Critical Sociology'. Alles andere - Abwarten vor allem - führt in eine Defensive, aus der es kein Entkommen gibt. Das mag für diejenigen, die es noch kommod auf Professuren oder unbefristeten Stellen aussitzen können, machbar sein. Aber der Verlust von ganzen soziologischen Instituten und Schwerpunkten prekarisiert den wissenschaftlichen Nachwuchs.

Vielleicht liefern die Paradoxien der Gegenwart eine interessante (Teil-)Antwort auf diesen ,Legitimationszwang', der durch Forschungsratings nur intensiviert wird. Genug Anregungen gibt es und die wurden vor allem in den vielen fokussierten ad hoc-Gruppen und einigen Sektionsveranstaltungen geboten. Ein genauer Blick auf die scheinbar trivialen Praxen der Lebenswelt fördert vielerlei Verunsicherungen und auch Kreativität der Akteure/innen zutage: Beispielsweise befasste sich Stephanie Duttweiler in ihrem Beitrag zur Veranstaltung „die paradoxen Körper" mit dem Paradoxon der alternden Gesellschaft, die ewig jung bleiben will. Während alte Menschen von allerlei Branchen intensivst beworben und auch medial inszeniert werden, verschwindet das Alter in seiner bisher bekannten Form. Jung wird mit den Imperativen der Gegenwart wie gesund, fit, mobil, flexibel, ungebunden gleichgesetzt; Alt-sein verkörpert hingegen die nicht verwertbaren und nicht optimierbaren Kehrseiten wie Verletzlichkeit, Abhängigkeit, räumliche Fixierung, Ruhe. Bei der Analyse entsprechender , anti-aging Kampagnen' wird klar: Alt werden? Gerne. Aber nur, wenn man dabei jung bleibt und „fit in die Kiste kommt“" (Duttweiler). Das mag lustig anmuten, doch wer mit über 35 Jahren z.B. nicht mehr verbeamtet wird (zu alt!), kriegt damit handfeste Probleme. Mit der Auflösung vermeintlicher Sicherheiten in Bezug darauf, was Alter ist, eröffnen sich der Soziologie hochinteressante und gesellschaftlich relevante Forschungsfelder. Gleiches lässt sich über sehr viele praxeologische Verunsicherungen sagen: Schönheit, Körperteile, Ethik, Finanzmärkte, Rationalität, Emotionen, Anerkennung, Entscheidungen, Karriere, Wissen, Geschlecht sowieso... Unsicherheit allerorten. Selbst die Krise ist in der Krise: „Alles in der Krise? Die Krise als Konzept zur Thematisierung von unsicheren Zeiten in Diskursen" so der Titel einer weiteren ad hoc-Gruppe.

Ich bin zuversichtlich, dass die Soziologie sich weiterhin gleichermaßen selbstbewusst wie jenseits von Pathos oder Verwertungspragmatismus der Unbestimmtheiten der sozialen Welt annimmt. Hinter dem erreichten Stand der (Selbst-)Reflexion, die sie in Teilen auszeichnet, kann sie jedenfalls nicht mehr zurück. Wir werden sehen, wie sich dies beim 35 . Kongress der DGS in Frankfurt am Main darstellt. 


\section{Literatur}

Bourdieu, Pierre (1976): Entwurf einer Theorie der Praxis, Frankfurt / Main.

Bröckling, Ulrich (2007): Das unternehmerische Selbst. Soziologie einer Subjektivierungsform, Frankfurt / Main.

Bude, Heinz / Heinz Willisch (Hrsg.) (2007): Exklusion. Die Debatte über die ,Überflüssigen', Frankfurt / Main.

Degele, Nina (2003): Happy together: Soziologie und Gender Studies als paradigmatische Verunsicherungswissenschaften, in: Soziale Welt 54, S. 9-29.

Dörre, Klaus (2008): Armut, Abstieg, Unsicherheit. Ein Essay, in: APuZ 33-34 / 2008, S. 3-6.

Gather, Claudia / Birgit Geissler / Maria S. Rerrich (Hrsg.) (2007): Weltmarkt Privathaushalt. Bezahlte Hausarbeit im globalen Wandel, Münster.

Klinger, Cornelia / Gudrun-Axeli Knapp (Hrsg.) (2008): ÜberKreuzungen. Fremdheit, Ungleichheit, Differenz, Münster.

Klinger, Cornelia / Gudrun-Axeli Knapp / Birgit Sauer (Hrsg.) (2007): Achsen der Ungleichheit. Zum Verhältnis von Klasse, Geschlecht und Ethnizität, Frankfurt / Main.

Lessenich, Stephan (2008): Die Neuerfindung des Sozialen. Der Sozialstaat im flexiblen Kapitalismus, Bielefeld.

Martschukat, Jürgen / Olaf Stieglitz (Hrsg.) (2007): Väter, Soldaten, Liebhaber: Männer und Männlichkeiten in der Geschichte Nordamerikas - ein Reader, Bielefeld.

Rosa, Hartmut (2005): Beschleunigung. Die Veränderung der Zeitstrukturen in der Moderne, Frankfurt / Main.

Villa, Paula-Irene (2006): Grübeln über einen diffusen Begriff - Zum Stand der Männerforschung, in: Soziologische Revue 2, S. 166-176.

Wissenschaftsrat (2008): Forschungsrating Soziologie, abgerufen am 16.12.2008 unter http://www.wissenschaftsrat.de/pilot_start.htm.

Prof. Dr. Paul-Irene Villa

Ludwig-Maximilians-Universität München Institut für Soziologie

Konradstraße 6

80801 München

paula.villa@soziologie.uni-muenchen.de 